\title{
Gliomas tipo astrocitoma pilocítico "atípico recurrente” y gen B-RAF: presentación de caso clínico, discusión
}

Glioma type pilocytic astrocytoma "atypical recurrent" and gen BRAF:Clinical case presentation, discussion

Fernando González Trujillo (1), Sergio Cafiero (2), Natalia Olaya (3), Alfonso Javier Lozano Castillo (4), Alfredo Ernesto Romero Rojas (5), Pedro José Penagos González (6), Camilo Zubieta Vega (7), Nicolás Gil (8), Gonzalo Melo Gómez (9)

\author{
RESUMEN \\ CONTRIBUCIÓN DE LOS AUTORES \\ Fernando González Trujillo, Autor \\ Sergio Cafiero, Autor \\ Natalia Olaya, Autor \\ Alfonso Javier Lozano Castillo, Autor \\ Alfredo Ernesto Romero Rojas, Autor \\ Pedro José Penagos González, Coautor \\ Camilo Zubieta Vega, Coautor \\ Nicolás Gil, Coautor \\ Gonzalo Melo Gómez, Coautor
}

El astrocitoma pilocítico (AP) es una neoplasia bien diferenciada, grado I OMS, que predomina en la infancia y es raro en la población adulta. Se reportan casos atípicos con una biología tumoral agresiva que continúan preservando la histología benigna o se transforman hacia gliomas de alto grado. Algunos estudios genéticos en esté subtipo de tumor referencian la activación de la vía MAPK/ERK a través de cambios en el gen BRAF. El objetivo del grupo es presentar un caso clínico representativo de un AP con evolución "atípica” y realizar una revisión actualizada desde la biología, genética, las posibilidades terapéuticas emergentes y exponer las controversias del tratamiento desde lo quirúrgico y las terapias complementarias.

PALABRAS CLAVES: gliomas, gen, mutación, deleción, atípico (DeCS).

1. Grupo de Neurología Clínica; profesional observador en Neurología Oncología; Instituto Nacional de Cancerología, Bogotá ESE; Clínica de Occidente, Santiago de Cali, Colombia

2. MD, MSc, Ph. D, jefe del servicio de Oncología Radioterápica; Centro Integral del Cáncer, Clínica del Occidente, Santiago de Cali, Colombia

3. MD, Ph. D,Grupo de Patología Oncológica. Instituto Nacional de Cancerología, Bogotá ESE, Colombia

4. Alfonso Javier Lozano Castillo,Grupo Radiología Oncología,Instituto Nacional de Cancerología, Bogotá ESE; profesor asociado, Universidad Nacional de Colombia, Bogotá, Colombia

5. Grupo de Patología Oncología, Instituto Nacional de Cancerología, Bogotá ESE,Bogotá, Colombia

6. Neurocirugía Oncología,coordinador Unidad de Neurocirugía-Oncología, Instituto Nacional de Cancerología, Bogotá ESE, Colombia

7. Neurocirugía Oncología, Instituto Nacional de Cancerología, Bogotá ESE;Clínica de Occidente, Bogotá, Colombia

8. Neurocirugía Oncología, Instituto Nacional de Cancerología,Bogotá ESE, Colombia

9. Neurología Oncología, Instituto Nacional de Cancerología, Bogotá ESE. Clínica Navarra, Bogotá, Colombia

Recibido 9/07/16. Aceptado: 18/07/17.

Correspondencia: Fernando González Trujillo, fernando.gonzaleztrujillo@gmail.com 


\section{SUMMARY}

The pilocytic astrocytoma (PA), formerly referred to as juvenile pilocytic astrocytomas, are WHO grade I tumors, that commonly occur during childhood and rarely in the adult population. Genetic studies of this tumor report an activation of the MAPK / ERK pathway through changes in the BRAF gene.

The aim of this article is to report a series of atypical PA cases with an aggressive tumor biology that continue preserving the benign histology or transformed into high-grade gliomas, and review the biology, genetics, and emerging therapeutic possibilities for these cases. And finally expose controversies from the surgical treatment and complementary therapies.

KEY WORDS: Gliomas, gene, mutation, deletion, atypical (MeSH).

\section{INTRODUCCIÓN}

Los astrocitomas pilociticos (AP) se presentan más en niños y adultos jóvenes, afectando principalmente áreas como el cerebelo, tallo cerebral, hipotálamo y vía óptica. Son considerados tumores benignos, con tendencia a formas quistes unio multilobulados con nódulo tumoral. Se manifiestan con un cuadro clínico de hipertensión endocraneana o signos asociados según el área comprometida. Hay excepciones entre los AP que pueden exhibir un comportamiento biológico agresivo "atípico y con recurrencias", la revisión de la literatura reporta casos con transformación hacia gliomas de alto grado tipo glioblastoma multiforme; la aplicación de estudios genéticos en este particular tipo de tumorreferenció la activación de la vía MAPK/ERK a través de cambios en el gen BRAF. Los AP son definidos como tipos agresivos y recurrentes no controlados con cirugías amplias, terapias adecuadas y con progresión dentro del primer año de seguimiento.

El grupo desea presentar una revisión de un caso clínico representativo de los tumores tipo AP con evolución "atípica" por su baja frecuencia de presentación; además, actualizar los conceptos desde la biología, la genética, las posibilidades terapéuticas emergentes y exponer las controversias del tratamiento desde lo quirúrgico y las terapias complementarias.

\section{Descripción del caso}

Paciente masculino, escolar, conocido en el Instituto Nacional de Cancerología, por cuadro clínico que inicióa la edad de 13 años con hiporexia, pérdida de peso no cuantificada, náuseas; 3 meses después diplopía derecha transitoria,parestesias en miembros inferiores; tuvouna evolución irregular, presentó convulsión clónica generalizada. El examen físico reveló signos de hipertensión endocraneana, fue trasladado a la unidad de cuidados intensivos donde le realizaron tomografía cerebral: se observó aumento del volumen del sistema ventricular con prominencia de las astas temporales de los ventrículos laterales, abombamiento del tercer y cuarto ventrículo; se complementó con reso- nancia cerebral, encontrando una masa supraselar central e izquierda, con extensión a la porción superior del seno cavernoso izquierdo, compresión de la hipófisis y tracción del tallo hipofisiario deformando el quiasma óptico. Porsu edad y los hallazgos referidos,se considerócomo probable diagnóstico un germinoma. Se analizaron los marcadores tumorales séricos gonadotropina coriónica $<0,5(0,00$ - 5), hormona folículo estimulante $0,1 \quad(2,1-18,5)$, hormona luteinizante menor de $1,0 \mathrm{mUi} / \mathrm{ml}(1,70-11,2)$ normales. Inicialmente se realizó biopsia con reporte por patología (institución externa) de unalesión tipo glioma de alto grado, probable astrocitoma. Permaneció en la unidad de cuidados intensivos pediátricos con evolución clínica estable; en el examen físico lo relevante fue una amaurosis, los potenciales evocados visuales demostraron un compromiso severo retino cortical. En el INC continuó su control. Tuvo deterioro clínicoy le realizaron imágenes de tomografía y resonancia cerebralesque mostraron"extenso proceso infiltrativo de probable origen neoplásico, con masa a nivel del hipotálamo, región selar y supra-selar; infiltración leptomeníngea de predominio basal, hidrocefalia activa con patrón comunicante". Los servicios tratantes considerarondescartar un proceso infeccioso crónico por la evolución clínica tórpida y los hallazgos atípicos en las imágenes; se realizaron estudios para hongos, tuberculosis, bacterias que fueron negativos. Se realizó la segunda resección de la lesión supraselar, el servicio de patología del INC revisó las placas histológicas iniciales (externas) y con las nuevas muestras obtenidas se concluyó como diagnóstico un astrocitoma pilocítico (OMS grado I) de la región supraselar (figuras1, 2). El paciente estuvo estable, la resonancia magnética de control evidenció "lesión basal frontotemporal izquierda con captación del medio de contraste, no se observómayor efecto de la masa local comparando con los estudios previos". Se dejó en seguimiento ambulatorio.

Los controles posteriores con resonancia cerebral simple y contrastada mostró "aumento de la lesión en la región hipotalámica que se extiende hacia la región temporal izquierda, asociado con lesión quística, captada de forma heterogénea el medio de contraste, con efecto compresivo 


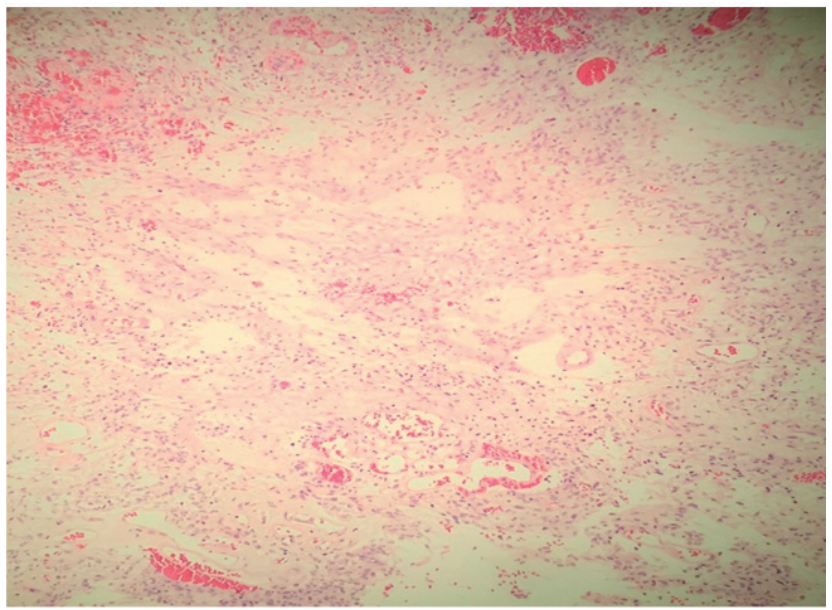

Figura 1. H\&E 10X. Se observa el patrón bifásico clásico del astrocitoma pilocítico con áreas microquistícas asociadas.

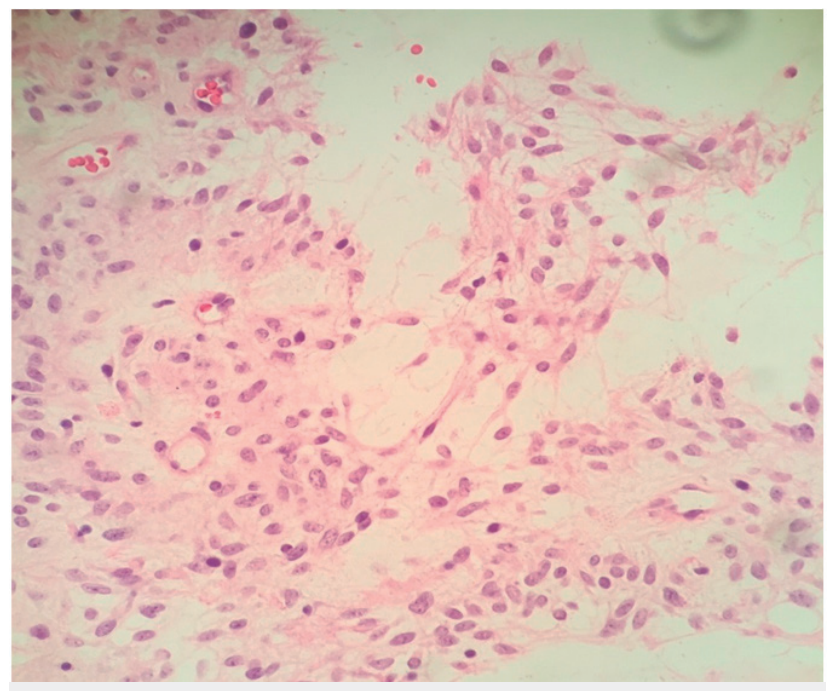

Figura 2. H\&E 40x. Se reconocen células bipolares sin atipia nuclear, mitosis ni necrosis.

y edema perilesional" (figura3); la Junta Médica interdisciplinaria de la institución, con la evidencia de aumento del volumen tumoral propuso cirugía, se colocó sistema derivativo en cuerno frontal derecho, con una buena evolución clínica posquirúrgica. La revisión patológica fue un astrocitoma pilocítico con diferenciación ependimaria, figura 4 (OMS 1), inmunohistoquímica: PGFA (proteína acida fibrilar glial) +, ema (antígeno membrana epitelial) +, sinaptofisina -, wt1 -, ki67 menor 1\%. Se estudió la mutación BRAF-V600E (se aplicó prueba comercial Cobas 4800 BRAF-V600 mutation test- laboratorio ROCHE) en las muestras de patología que fue negativo. La Junta Médica propuso tratamiento comple-

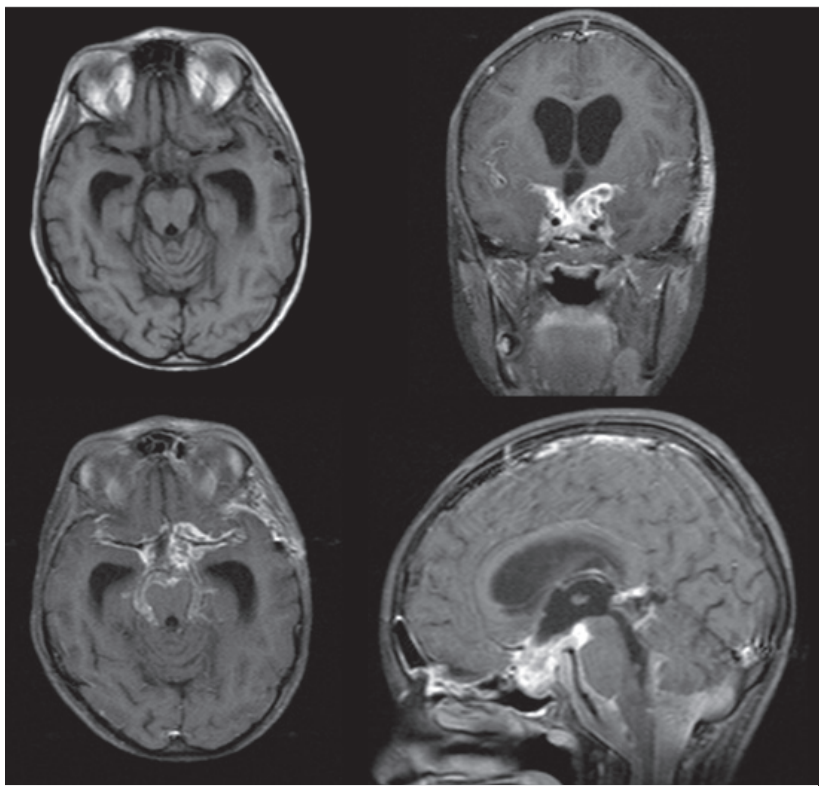

Figura 3. IRM contrastada, marzo de 2009: extenso proceso infiltrativo leptomeníngeo selar, supraselar y en las cisternas basales de probableorigen neoplásico con tumor conocido enhipotálamo izquierdo. Aumento en el volumen de los ventrículos laterales, con patrón de hidrocefalia activacomunicante.

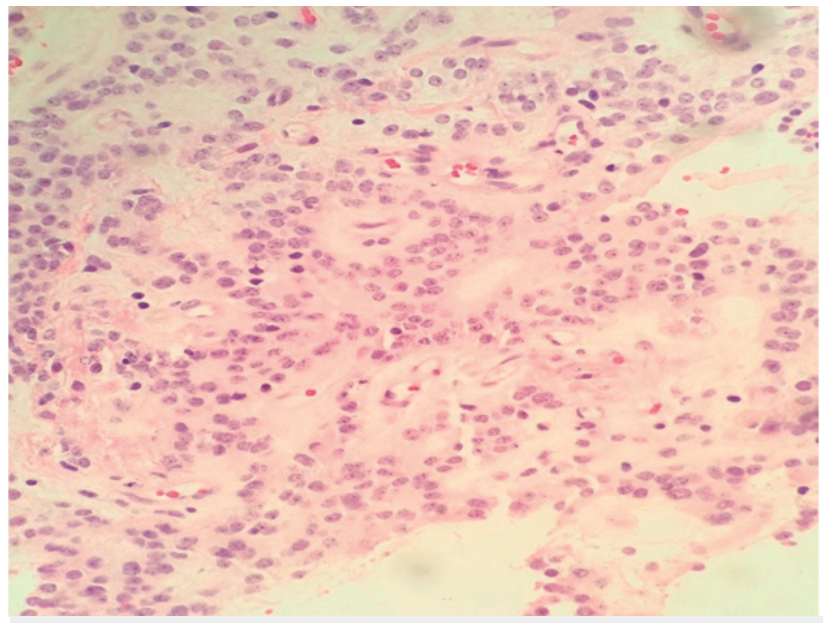

Ffigura 4. H\&E 10x. Se identifica diferenciación ependimaria con formación de pseudorosetas perivasculares y esbozo de rosetas ependimarias.

mentario con radioterapia. Se programó radioterapia con fotones de $6 \mathrm{mv}$ técnica conformacional $3 \mathrm{dcrt}$ para irradiar lesión tumoral con margen, dosis de 2 Gy día hasta 50 Gy y posterior reducción de campo para refuerzo hasta $60 \mathrm{~Gy}$.

Fue valorado por endocrinología sin evidencia clínica y por laboratorios para un déficit hipofisario (anexamos resultados: t4l:1,44ng/dl, FSH: $3,78 \mathrm{mUi} / \mathrm{ml}$, testosterona total: 
4,15ng/ml (n), cortisol: $396 \mathrm{nmol} / \mathrm{l}(14,4 \mathrm{ug} / \mathrm{dl})$, prolactina: $5,7 \mathrm{ng} / \mathrm{ml}$. Siguió en controles ambulatorios, presentórecidiva de síntomas vertiginosos con los cambios de postura, el examen físico destacó el severo compromiso visual y atrofia óptica. Requirió hospitalización por desorden metabólico tipo hiponatremia, síndrome convulsivo (recibió fenitoína), disartria yse trasladó a la unidad de cuidado intensivo. La imagen de TC cerebral de control evidenció edema frontal bilateral y temporal izquierdo, sangrado interpeduncular e intraventricular (tercer y cuarto ventrículos) y catéter de derivación en el asta frontal derecha (figura 5). El grupo neuroquirúrgico consideró no realizar otra cirugía. Presentó complicaciones con un estado convulsivo refractario, estado séptico, choque hemodinámico, requirió soporte vasopresor y ventilatorio y falleció.

\section{Biológica tumoral y oncogénesis - gen BRAF}

La biología molecular y genómica por alta resolución aplicada en los tumores ha permitido mejorarla información de los gliomas, se identificaron vías celulares citoplasmáticas importantes como las MAPK/ERK y PI3K/AKT, se amplificaron los genes que codifican los receptores tirosinquinasas (EGFR, PDGFRA), delecciones, mutaciones en genes supresores tumorales (tp53, p16, PTEN).

Los gliomas de bajo grado (GBG) presentan alteraciones genéticas asociadas con el desarrollo, histología, comportamiento clínico agresivo; ejemplos:con el subtipo astrocitoma pilocítico (AP) se reporta la activación de la vía MAPK/ ERK a través de cambios en el gen BRAF,por una duplicación aleatoria en el dominio "kinasa" que genera una fusión génica referenciada como “BRAF:KIAA1549”, eventos de fusión-duplicación génica que involucran RAF1 y SRGAP3, la mutación BRAFV600E (produce activación constitutiva de BRAF). Los AP relacionados con neurofibromatosis tipo 1 (NF1) contienen la inactivación homocigota del gen NF1 vinculado con la activación de la vía MAPK/ERK (1).

El BRAF es mediador activo en la vía de señalización RAS-RAF-ERK kinasa (MEK)-señal extracelular regulador de kinasas (ERK)- proteína kinasa asociada a microtúbulo (MAP); se vincula con diversas funciones celulares como lamediación o paro del ciclo celular, proliferación celular, diferenciación celular y apoptosis (2). Los GBG localizados en la vía óptica en pacientes con neurofibromatosis tipo 1 son evidencia importante del rol que cumple la activación de la vía RAS-MAPK, que tiene una alta tasa de presentación entre estos tumores. El grupo de Pfister y colaboradoresevidencióla duplicación en el cromosoma 7q34 que contiene al gen BRAF, es un "gen corriente abajo" en la vía RASMAPK (3). La activación de estavía en los GBG no explica completamente el proceso benigno en estos tumores y la tendencia a detener su crecimiento; surge el concepto de

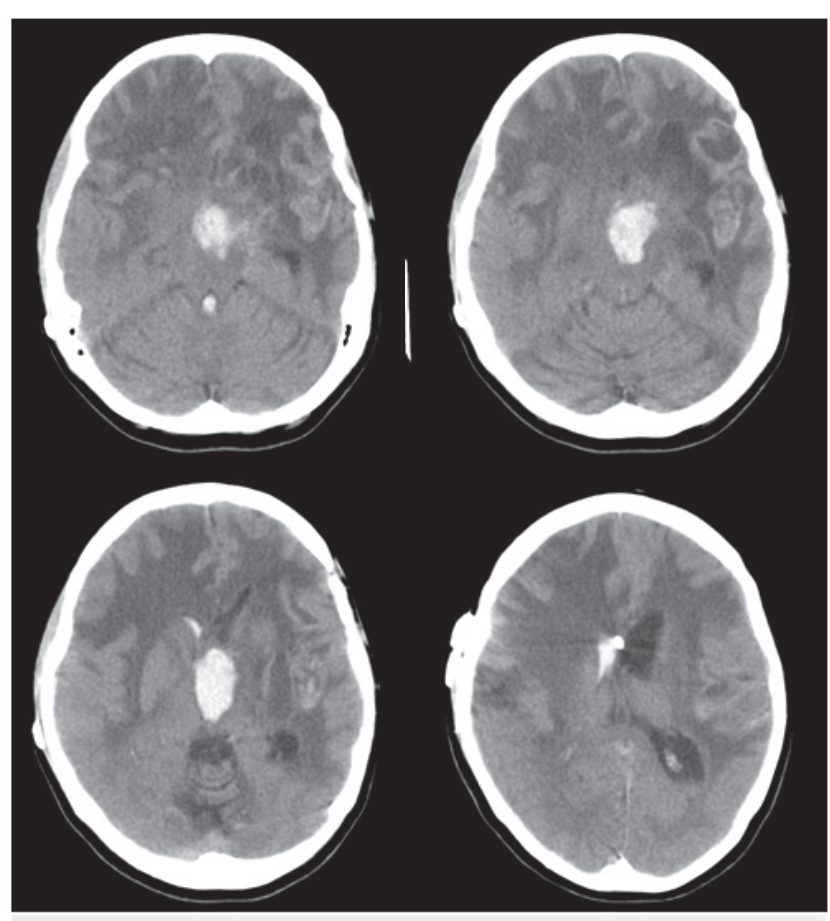

Figura 5. TC cráneo simple, junio 2012: edema vasogénico comprometiendo los lóbulos frontales y el temporal izquierdo, con áreas sangrado en cisterna interpeduncular, tercer y cuarto ventrículos.

"oncogén inductor de senescencia" para explicar porque es necesario la activación de RAF para producir el retroceso del crecimiento tumoral (3). Bartkova y colaboradores demostraron en tumores de colon, seno, vejiga que esta vía es precancerosa, no encontraron alteración concomitante en genes supresores tumorales como p53, RB; hubo tendencia de las células cancerosas hacia la senescencia y apoptosis (3). Dado, que los tumores gliales de bajo grado en la población pediátrica (que son diferentes a los de adultos) expresan poco o ningúncambio en los genes TP53 y RB, se postula que por el mecanismo "oncogén inductor de senescencia"se explica la poca progresión hacia formas malignas yhasta darse la regresión tumoral espontanea (3).

BRAF (figura 6) como componente de la vía intracelular - proteína-quinasa activadora de mitogenesis (MAPK) - inicia la activación de un receptor transmembrana tirosinaquinasa, ocurren procesos de fosforilación para activar Ras, luego Raf-quinasa, MEK 1/2 y la posterior activación del complejo de transcripción ERK1/2 $(3,4)$. El gen Raf tiene 3 isoformas: A-Raf, B-Raf, yC-Raf, que siguen un mecanismo idéntico de función donde un dominio regulador N-terminal inhibe el dominio C-terminal, la unión de Ras al dominio N-terminal produce liberación del bloqueo normal. Cada isoforma de Raf en forma independiente puede fosforilar a MEK, se reporta una mayor frecuencia 


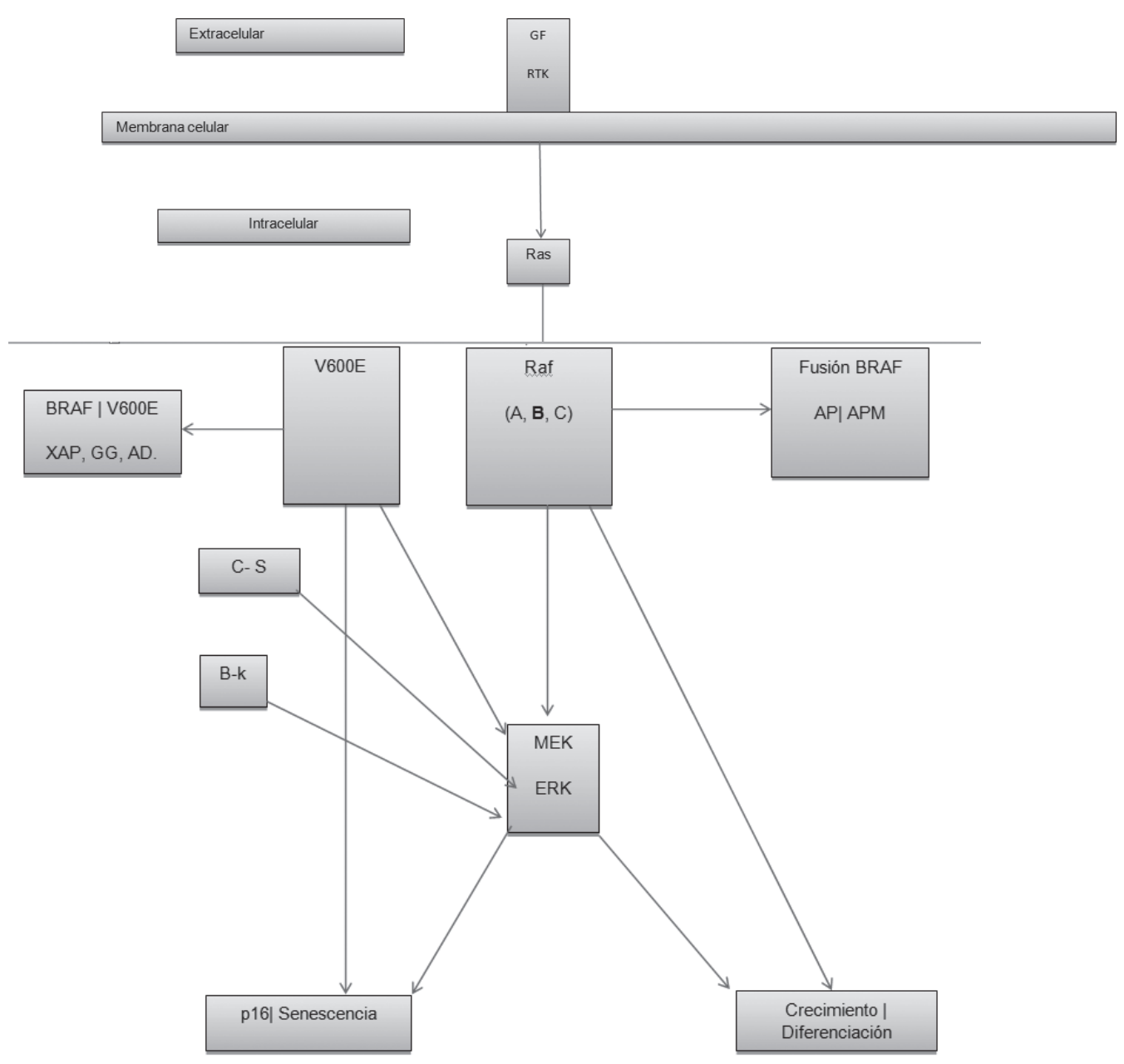

Figura 6. Vía de señalización MAPK y alteraciones en B-Raf: normalmenteRas, activa las proteínas Raf, la proteína B-Raf es la más potente activadora de MEK promoviendo la división, sobrevida celular. Con las alteraciones genéticas tipo fusión B-K, C-S, la mutación V600E en B Raf activan MEK sin mediación de Ras, lo que produce crecimiento tumoral. Cuando la proteína del "punto de chequeo" p16 está intacta, la sobreactivación de la vía MAPK conduce a la senescencia tumoral. Asociado con los hallazgos histológicos, la presencia de fusión BRAF sugiere tumores tipo PA, PMA. EI detectar B-Raf V600E apoya tumores tipo PXA, GG o DA.

GF:factor de crecimiento; RTK:receptor tirosin kinasa; XAP: xantoastrocitoma pleomórfico; GG:ganglio-glioma; AD: astrocitoma difuso; AP: astrocitoma pilocítico; APM: astrocitoma pilomixoide. (Adaptado de referencias 4, 5, 9).

con la mutación B-Raf porque B-Raf tiene solo 2 sitios de activación quinasas y A-Raf, B-Raf tienen 4. Además, el B-Raf es un activador más potente de MEK “corriente abajo" en la vía de señalización y el potencial oncogénico es mayor comparado con las otras isoformas Raf (4).

En los gliomas las alteraciones en BRAF más reportada son re-arreglos génicos y fusiones. El cromosoma 7q34 que contiene el gene BRAF, tiene "puntos de ruptura" que facilitan producir una proteína de fusión constitutivamente activa (3- 5). La mayoría de las mutaciones para BRAF son anti-sentido, hay una con intercambio entre los aminoácidos valina por glutamato en la posición 600 (la nomenclatura es conocida como BRAFV600E), la frecuencia de presentación es variable con reportes bajos como el 5,9\% hasta valores altos como de $60 \%$ con tumores tipo xantoastrocitoma pleomórfico (PXA), en la serie reportada por Dias- Santagata y colaboradores, Schindler y colaboradoreses de 18\% para ganglio-gliomas (GG), y 9\% en astrocitomas piloci- 
González Trujillo F, Cafiero S, Olaya N, Lozano Castillo AJ, Romero Rojas AH, Penagos González PJ, Zubieta Vega C, Gil N, Melo Gómez M.

ticos (PA). Otros subtipos tumorales con reportes de la mutación son: oligodendrogliomas, tumores ependimarios y glioblastoma multiforme. La mutación BRAFV600E no se ha reportado, hasta ahora en los subtipos tumorales (por referencia estadística) tipo meduloblastoma, tumor neuroectodérmico primitivo, tumor rabdoideteratoide, neurocitoma central ytumor neuroepitelial disembrioplástico (2).

Los reportes de grupos de trabajo ( Lin A y col., Jones y col. Collins y col.) coinciden en la propuesta que la proteína producto de la fusión KIAA1549:BRAF (duplicación aleatoria) se transcribe desde el promotor KIAA154 (3-5), que la transformación neoplásica se sostiene con niveles balanceados del producto, se verificó que los niveles bajos de estelograban mantener el proceso de malignidad; en oposición, se encontró que el exceso en los niveles delaproteína mutante BRAF puede interferir con los procesos de progresión maligna y promover la activación del oncogén hacia la senescencia en la célula tumoral. La fusión KIAA1549:BRAF y su expresión guarda relación con la localización anatómica del tumor, los grupos de trabajo reportan más la expresión con el subtipo tumoral astrocitoma pilocítico (AP) localizado en la vía óptica o la fosa craneal posterior, en contraposición, el grupo del Dr. Lin y colaboradores no la identificó en su serie de casos pediátricos. Con las variables estado de la fusión y edad, se identificó que la población con mayor edad tenía una expresión más baja en relación con la población joven. Otras fusiones que se reportan con los AP son: la 1Y16/11Y18 relacionada con localización infratentorial, la 1Y15/11Y18 con localización supratentorial $(4,5)$.

Las alteraciones génicas en Raftipo mutación, fusión lo hacen constitutivamente activo, estado que permite activar directamente a MEK sin la intervención "corriente arriba" de RAS fosforilado. Encontrar mutaciones y fusiones expresadas en el mismo tumor es raro, hay reportes decasos en adultos donde la mutación V600E o la fusión en BRAF coexisten con otras mutaciones tipo IDH1/2(5). Se destaca que la coexistencia con la deleción p16 en casos de población pediátrica es un marcador de pronóstico adverso, la pérdida de p16 inhibe que BRAF induzca senescencia del tumor (5). B-Raf-V600E en población pediátrica se asocia con sobrevida de libre de progresión corta, mientras la presencia de la fusión B-Raf se relaciona con sobrevida libre de progresión más prolongada $(4,5)$.

La mutación V600E en el gen BRAF puede servir para fines de diagnóstico, de pronóstico y tratamiento; la experiencia obtenida con el medicamento vemurafenib (es selectivo y potente inhibidor de brafv600e) en pacientes con melanomaavanzado que tienen la mutación,logrando resultados favorables en sobrevida y sobrevida libre de progresión que motivo ampliar su aplicación en los gliomas de bajo grado que la expresen y se han referenciado resultados positivos en varios casos de pacientes (4).

\section{Astrocitoma pilocítico "atípico": generalidades}

El astrocitoma pilocítico (AP) es una neoplasia bien diferenciada entre los tumores astrocíticos, se presenta en el 40\% de los casos en la infancia y es infrecuente en la población adulta. El pronóstico de sobrevida es favorable, se reportansolo con la resección quirúrgica amplia o total, promedio a 5 años hasta de $95 \%$. Se reportan casos excepcionales de pacientes con progresión clínica y recurrencia tumoral hasta en el $20 \%$ de los datos según los diferentes grupos de trabajo, con índices altos de morbilidad, mortalidad y sin hallazgos por patología de cambios por anaplasia tumoral. Los AP son definidos como tipos agresivos y recurrentes no controlados con cirugías amplias, terapias adecuadas y con progresión dentro del primer año de seguimiento (6-8).

Hay factores determinantes en el paciente para una buena evolución como: la extensión de la resección quirúrgica, la localización del tumor (tumores infratentoriales tienen una excelente sobrevida libre de progresión de 100\% a 5 años), los tumores quísticos comparados con los sólidos tienen mejor pronóstico (independiente de la localización), y la recurrencia y uso de radioterapia (8).

En patología se define un tumor como AP, según los criterios de la OMS, por la presencia de un patrón bifásico de células bipolares compactadas y células multipolares, pérdida de textura asociadas con fibras de Rosenthal y cuerpos granulares eosinofílicos; la inmunoreactividad positiva para el marcador proteína acida fibrilar glial (GFAP) se detecta más en las células bipolares, el índice de mitosis Ki-67 es generalmente menor a 1\% (8). La "fusión génica" BRAF:KIAA1549 (B-K) es común pero no específica, la expresan otros gliomas de bajo grado tipo astrocitomas difusos y pilomixoide. La Dra. Hawkins y colaboradoresmostró que la fusión $\mathrm{B}-\mathrm{K}$ fue común encontrarla en los $\mathrm{AP}$ localizados en la línea media, donde la resección completa es imposible (6, 9-11).

No hay consenso en el plan de manejo de pacientes con recurrencia, para algunos grupos la intervención depende del volumen residual tumoral valorado por resonancia magnética posoperatoria, estado clínico y funcional del paciente, riesgo del procedimiento quirúrgico (8). Los grupos quirúrgicos tienen controversia respecto a la extensión de la cirugía inicial, el Dr. Stuer y colaboradores reportóla serie de 44 pacientes donde la extensión de la resección fue un predictor significativo para la sobrevida libre de progresión y una mejor sobrevida total (12).

No existen guías definidas de tratamiento para la fase inicial posoperatoria, las directrices no son claras con los 
resultados obtenidos en pacientes que recibieron tratamiento complementario en primera instancia o cuando hubo progresión tumoral. Tradicionalmente, los pacientes se observan o son referidos para radioterapia adyuvante cuando hay residuo tumoral (12). El Dr. Ishkanian y col. encontró un porcentaje alto $-60 \%$, de pacientes en riesgo de progresión tumoral a 5 años, destacó que la radioterapia posoperatoria en estos casos puede retardar la progresión en forma significativa, pero no impactala sobrevida total; la recomiendan en los AP localizados en "áreas cerebrales elocuentes" $(12,13)$.

Los pacientes sometidos a radioterapia complementaria presentaron progresión dentro de los 5 años siguientes, la recaída (se aclara que se confirmó por patología y correspondió con $\mathrm{AP}$ ) puede ocurrir dentro del volumen de campo irradiado o con diseminación leptomeníngea (13). Cuando se comparó la radioterapia convencional y la radiocirugía no hubo diferencias, el Dr. Mansur y col. trataron pacientes con radiocirugía, reportando que mejoró la sobrevida libre de progresión a 5 años en un 80\% comparado con el 66\%, observado con radioterapia convencional, lo cual según sus resultados no fue estadísticamente significativo. Entre los pocos trabajos realizados solo agrupando AP, se tiene el realizado por el Dr. Boethius y col. que evidenció en 19 pacientes, un $100 \%$ de sobrevida libre de progresión a 5 años cuando se aplicó radiocirugía tipo Gamma knife. El grupo de la universidad de UCLA en California reportó la experiencia en 12 pacientes con AP residual, 9 pacientes recibieron radioterapia estereotáxica en dosis promedio de 50,4 Gy, recomiendan que fue segura y efectiva para el control de progresión de un AP residual $(13,14)$.

En losniños muy jóvenes donde la radioterapia tiene alta toxicidad, la quimioterapia emerge como alternativa para postergarla en este grupo poblacional, la eficacia no es clara(13). El Dr. Gururangan y col. reportó pacientes con AP tratados con temozolamida con sobrevida libre de progresión a 4 años solo del 31\% (13). Los reportes de radio-quimioterapia en AP del estudio "Children cáncer Group CCG-945” con astrocitomas de bajo grado, donde incluyeron $19 \mathrm{AP}$, evidenciaron una sobrevida libre de progresión de 68\% que no superó otros esquemas (13).

\section{DISCUSIÓN}

Los AP con evolución "atípica" son casos excepcionales que progresan dentro del primer año y tienen altos índices de mortalidad, el caso clínico presentado esun ejemplo representativo que debe reportarse en la literatura.

Las lesiones con distribución difusa y un infiltrado leptomeníngeo que comprometía la pared del seno cavernoso izquierdo y la región hipotalámica, incluido el tracto óptico, tienen entre los diagnósticos diferenciales patologías infec- ciosas por bacterias, hongos, tuberculosis, etc,descartándose por los estudios extendidos que fueron negativos.

Se logró demostrar por resultados de patología (muestras obtenidas en diferentes estadios y por cirugía abierta) la histología benigna del tumor con un índice de proliferación Ki67 menor al 1\%, lo que evidenció que el tumor nunca progresó hacia una forma de anaplasia.

El estudio de la mutación génica BRAF-V600E fue negativo, y se ha reportado en muchos tumores extracerebrales y últimamente en gliomas de bajo y alto grado. Adicionalmente, consideramos se debió analizar la "fusión génica” BRAF:KIAA1549 (B-K) por ser la más reportada en este tumor, pero, no se tenían los recursos.

El tumor localizado en la línea media dificultó la resección quirúrgica amplia, es un marcador determinante para las variables de sobrevida libre de progresión y sobrevida total, los mejores resultados se reportan con la localización infra-tentorial y la resección amplia con mínimo residuo tumoral. Se observó en el paciente un periodo aproximado de 18 meses con una evolución estacionaria sin progresión, que es lo esperado para un tumor benigno de tipoglial; como lo referencia la literatura para los tumores tipo AP atípicos y recurrentes,la evolución posterior del paciente fue hacia el deterioro clínico y progresión tumoral con diseminación leptomeníngea que se evidenció por imágenes de resonancia $(13,15)$.

El grupo neuroquirúrgico de la institución en los gliomas de bajo grado considera que la resección total es primordial para evitar la recidiva temprana y en muchos casos constituye la única terapia requerida por el paciente, evitando los tratamientos complementarios con radioterapia o quimioterapia. Este caso en particular presentó una evolución diferente a lo observado con los tumores de bajo grado, tornándose difuso y agresivo, se consideró como un tumor anaplásico y se concluyó que la primera resección fue parcial y no se obtuvo una muestra significativa o representativa de las áreas de mayor anaplasia tumoral, lo que motivó la segunda cirugía realizada en el INC.Hubo dificultad para realizar la resección completa por la distribución difusa y por la biología tumoral agresiva del tumor, se definió como terapia complementaria la radioterapia, acorde con los reportes de la literatura sobre la quimioterapia. Los resultados logrados fueron malos, sinlograr contener el crecimiento del tumor. Los tratamientos complementarios a la cirugía no están bien definidos, deben considerarse en fase temprana a la cirugía cuando queda remanente importante del tumor y la decisión respecto a la radioterapia, la concomitancia de radio-quimioterapia debe ajustarse a la condición clínica del paciente. Las terapias emergentes contra "objetivos moleculares específicos" como la mutación génica BRAF-V600E están en proceso de consolidarse en el campo de los gliomas 
González Trujillo F, Cafiero S, Olaya N, Lozano Castillo AJ, Romero Rojas AH, Penagos González PJ, Zubieta Vega C, Gil N, Melo Gómez M.

de bajo grado, se requiere ganar experiencia con los casos de gliomas "atípicos recurrentes" o que sufren diferenciación hacia tumores anaplásicos tipo glioblastomas, para los cuales serán una importante opción terapéutica $(4,16,17)$.

\section{CONCLUSIÓN}

Los astrocitomas pilociticos (AP) son considerados clásicamente como tumores benignos y para la mayoría de casos la resección tumoral amplia logra ser curativa. Pero, los casos de AP con evolución atípica, con o sin transformación hacia gliomas de alto grado (15) son referenciados cada vez más por los diferentes grupos de trabajo; entonces, se hace necesario avanzar para asociar la genética con los estudios de patología tradicional y mejorar el conocimiento de la biología tumoral en estos tumores. Se tendrá una información relevante que permitirá el desarrollo de nuevas terapias "diana" para aplicar en paralelo a los tratamientos complementarios tradicionales.

\section{Conflictos de intereses}

Los autores manifiestan no tener conflictos de intereses en este estudio.

\section{REFERENCIAS}

1. Rodriguez EF, Scheithauer BW, Giannini C, Rynearson A, Cen L, Hoesley B ET A. PI3K/AKT pathway alterations are associated with clinically aggressive and histologically anaplastic subsets of pilocytic Astrocytoma. Acta Neuropathol. 2011;121(3):407-20.http://dx.doi.org/10.1007/s00401-0100784-9

2. Myung, Hwajin C, ChulKee P, Seung-Ki K, Se-Hoon L, Jae K.Analysis of the BRAFV600E Mutation in Central Nervous System Tumors.Transl Oncol.2012;5(6):430-36.http://dx.doi. org/10.1593/tlo.12328

3. Hawkins CE, Walker E, Nequesha M. BRAF-KIAA1549 fusion predicts better clinical outcome in pediatric low grade astrocytoma. Clin Cancer Res.2011;17(14):4790-8.http://dx.doi. org/10.1158/1078-0432.CCR-11-0034

4. Horbinski C.To BRAF or Not to BRAF: Is That Even a Question Anymore?.J Neuropathol Exp Neurol. 2013;72(1):2-7. http://dx.doi.org/10.1097/NEN.0b013e318279f3db

5. Lin A, Rodriguez FJ, Karajannis MA, Williams SC, Legault G, Zagzag D, et al. Identification of 2 Novel KIAA1549:BRAF Fusion Variants. J Neuropathol Exp Neurol. 2012; 71:66-72.

6. Rodriguez FJ, Scheithauer BW, Burger PC, Jenkins S, Giannini C. Anaplasia in Pilocytic Astrocytoma Predicts Aggressive Behavior. American Am J Surg Pathol.2010;34(2):147-60. http://dx.doi.org/10.1097/PAS.0b013e3181c75238.

7. Johnson DR, Brown PD, Galanis E, Hammack JE. Pilocytic astrocytoma survival in adults: analysis of the Surveillance, epidemiology, and end results program of the National Cancer Institute. J Neurooncol. 2012;108(1):187-93.http://dx.doi. org/10.1007/s11060-012-0829-0

8. Bilginer B, Narin F, karlioguz K, Uzun S, Soylemezoglu F, Akalan N. Benign cerebellar pilocytic astrocytomas in children. Turk Neurosurg. 2011; 21(1):22-26.

9. Hawkins C, Walker E, Mohamed N, Zhang C, Jacob K, Shirinian M, et al. BRAF-KIAA1549 fusion predicts better clinical outcome in pediatric low grade astrocytoma. Clin Cancer Res. 2011;17(14):4790-8.http://dx.doi.org/10.1158/1078-0432. CCR-11-0034

10. Schindler G, Capper D, Meyer J, Janzarik W, Omran H, Mende $\mathrm{CH}$, et al. Analysis of BRAF V600E mutation in 1320 nervous system tumorsreveals high mutation frequencies in pleomorphic xanthoastrocytoma, ganglioglioma and extra-cerebellar pilocytic astrocytoma.ActaNeuropathol.2011;121(3):397-405. http://dx.doi.org/10.1007/s00401-011-0802-6

11. AlShakweer W, Alwelaie Y, Mankung AM, Graeber MB. Bone marrow-derived microglia in pilocyticastrocytoma.Front Biosci (Elite Ed). 2011; 3:371-79.

12. Ishkanian A, Laperriere NJ, Xu W, Millar BA, Payne D, Mason W. Upfront Observation versus radiation for adult pilocytic astrocytoma. Cancer. 2011;117(17):4070-79.http://dx.doi. org/10.1002/cncr.25988

13. Mansur DB , Rubin JB , Kidd EA, King AA , Hollander AS , Smyth MD, et al. Radiation therapy for pilocyticastrocytomas of childhood.Int J Radiat Oncol Biol Phys.2011;79(3):829-34. http://dx.doi.org/10.1016/j.ijrobp.2009.11.015

14. Lizarraga KJ, Gorgulho A, Lee SP, Rauscher G, Selch MT,DeSalles AF. Stereotactic radiation therapy for progressive residualpilocytic astrocytomas. J Neurooncol. 2012;109(1):12935.http://dx.doi.org/10.1007/s11060-012-0877

15. McLendon RE, Adesina AM.Pathology of Expansile Astrocytomas [Internet]. Updated: Sep 15, 2015. Disponible:emedicine. medscape.com/article/1780937-overview

16. Kleinschmidt BK, Aisner DL, Birks DK,Foreman NK. Epithelioid GBMs show a high percentage of BRAF V600E mutation. Am J Surg Pathol. 2013;37(5):685-98.http://dx.doi. org/10.1097/PAS.0b013e31827f9c5e

17. Robinson GW, Orr BA, Gajjar A. Complete clinical regression of a BRAF V600E-mutant pediatric glioblastoma multiforme after BRAF inhibitor therapy. BMC Cancer. 2014; 14:258-62. https://doi.org/10.1186/1471-2407-14-258 